\title{
CHOOSING BY ADVANTAGES FOR THE SELECTION OF A NEW MEMBER OF THE PROJECT TEAM
}

\author{
Anthony F. Paucar-Espinoza ${ }^{1}$, Andrews A. Erazo-Rondinel ${ }^{2}$, and Seiko Yong- \\ Zamora $^{3}$
}

\begin{abstract}
The construction industry works through projects; each project needs people who make its realization possible, and these people relate to each other, forming work teams. Thus, there is an important relationship between the projects and the team members of a construction project, who must be selected based on competencies that allow them to satisfactorily perform their role in the project and thus contribute to the project's success. This research aims to provide a systematic approach while also providing decisionmakers with best practices by demonstrating the application of the Choosing By Advantages (CBA) system tabular method in selecting a member of the project team. To this end, the research begins with a bibliographic compilation to consolidate the main factors that allow us to choose a new member of the project team. Later, the team is trained in the CBA system. The choice is determined by applying the Tabular CBA method to support a collaborative virtual platform and a remote communication program. Finally, the team decided and chose the new member to be part of the project team in the Project Control area.
\end{abstract}

\section{KEYWORDS}

Choosing by Advantages, project team, multi-criteria decision analysis, CBA tabular method, project controls.

\section{INTRODUCTION}

The construction industry works through projects (Campero \& Alarcon, 2003); each project needs people to make it possible, and these people interact with each other forming work teams (Fong and Lung, 2007). Thus, work teams are part of organizations that allow individuals to satisfy different needs: emotional, spiritual, intellectual, economic. Ultimately, organizations exist to achieve goals that isolated individuals cannot achieve due to their limitations. Across organizations, the last limitation to achieve many human goals is working efficiently as a team (Chiavenato, 2009). Therefore, there is an important relationship between the organization and the human resource. Therefore, it is necessary to have a more prepared staff, who adapt more quickly to modern technology, proactive,

Researcher, Faculty of Civil Engineering, Universidad Nacional de Ingeniería, Lima, Peru, apaucare@uni.pe, orcid.org/0000-0002-5369-1584

2 Teaching Assistant, Faculty of Civil Engineering, Universidad Nacional de Ingeniería,, Lima, Peru aerazor@uni.edu, orcid.org/0000-0002-5639-573X

3 Planner, Consorcio Sihuay, Ancash, Peru, seiko.yong@consorciosuyay.com, orcid.org/0000-00030635-374X 
and know-how to interpret what changes are generating (García and Tantalean, 2012). That is why the selection of project team participants is an important decision. Usually, intuitive decision-making is carried out, that is to say, to perform a rapid recognition of patterns and select an alternative based on the stored memories (Wilson, 2003). Alternatively, consider cost as the predominant factor in decision-making processes, or even little or no other stakeholders' participation (Ding and Parrish, 2019). In this way, CBA could help discern the relative value between applicants better and know the skills gap to develop to occupy a specific role in construction projects.

Thus, the following research aims to develop the CBA application to select a project control team member. For this, the investigation begins with a literary review of the selection process that construction companies follow, how the project control areas, and the lean profile for construction projects are composed. With this information, the tabular CBA method's application is carried out, and one of the three candidates is selected for the project control position.

\section{RESEARCH METHOD}

The following research is part of a case study and details the application of CBA in selecting a member for the team of a Hospital project in Peru (Figure 1). For this, a literary review of the recruitment and selection process of team members, roles of the Project Control area, and the competencies required for a lean professional to perform construction projects are initiated. The factors are selected, taking into account the lean competence developed by Pavez \& Alarcon (2007). With the factors already defined, the project team is trained in the CBA system, the CBA Tabular method, and the steps of its application. Finally, the Tabular CBA method's application is illustrated in the selection of a new member of the Project Control area team in a hospital building project in Peru.

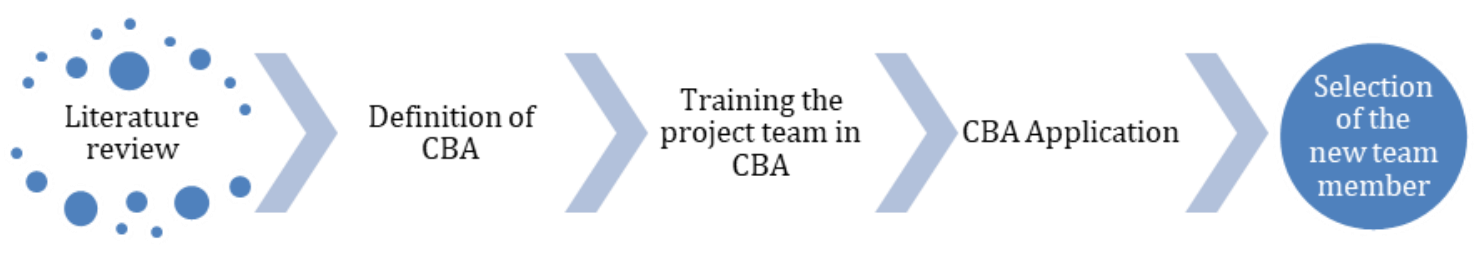

Figure 1: Stages of research

\section{THEORETICAL OVERVIEW}

In an engineering and construction company, the key processes are based on obtaining and executing projects, while the other areas support them. Recruitment and selection are within the functional area of Human Resources in the company's central office; its objective is to satisfy the demand for personnel from the different areas that make up the company and the projects, according to specific profiles requested (Castellano, 2013). According to Castellano (2013) the recruitment and selection process in a company whose projects are developed in the Construction and Engineering sector has the following steps (Figure 2):

1. Launch of the admission requirement: The search begins once the request has been made by the client, this requirement is originated from 3 sources, the Staffing Plan (Project Personnel Plan), Individual Requirements and Proposed Candidates.

2. Internal Search: Then, the requested requirements are searched in the internal database, the search is made based on the defined profiles and the candidates are filtered 
towards the one that suits the requested position. In this database, personnel information is stored soon to leave a project, those that have been saved by previous applications and proposals. If there is no candidate that meets the requested requirement, a search is carried out in external sources.

3. External Search: The process, therefore, continues with the definition of the profile of the requested requirement and the profile survey, managing to have the information of the personnel that is required in detail (what is defined in step 1 is made explicit but this time in greater detail for an optimal external search). Subsequently, the requirement is published in external sources (via the web, publications in specialized magazines, other external sources). In this way, the Curriculum Vitae of the candidates who apply are filtered. This is how the approval of the candidates sent is expected to cite them to Evaluations or continue with the search in external sources. Once this process is finished, the second phase of the process, that of Selection, continues.

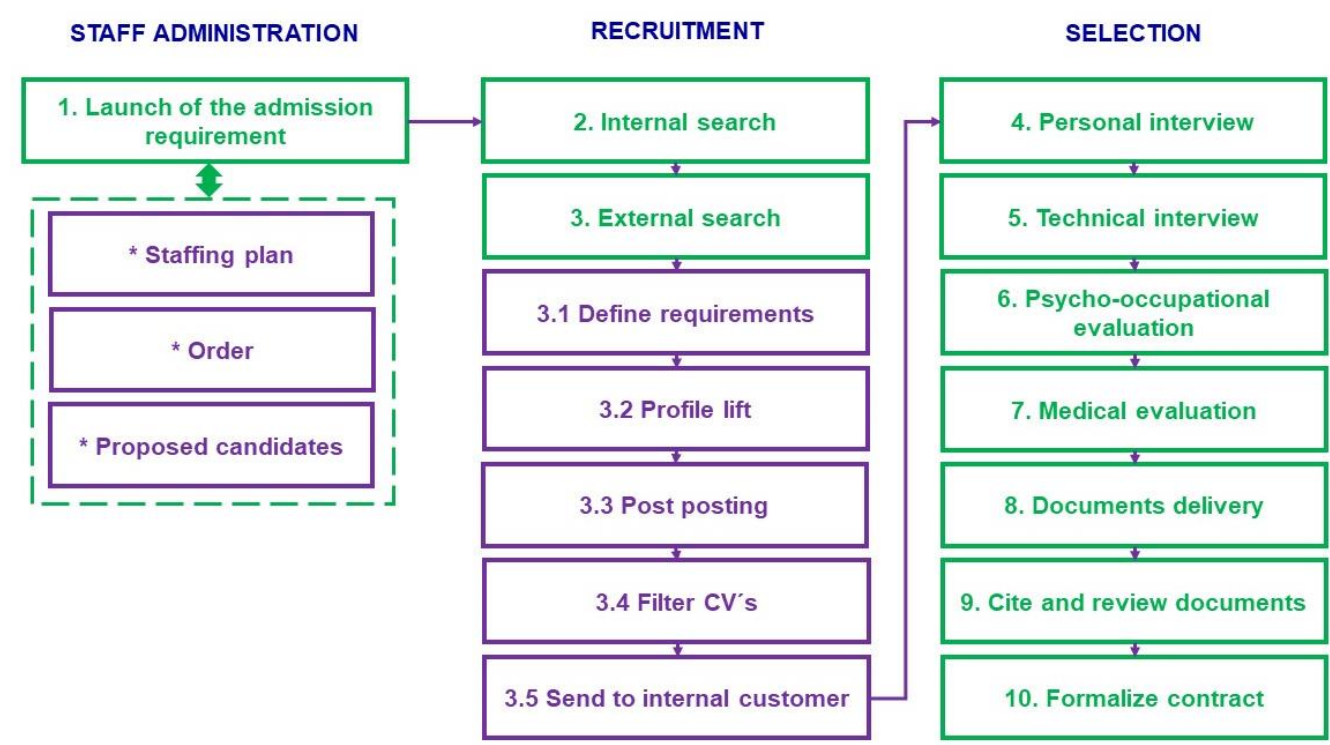

Figure 2: Selection Process in Construction Projects. Adapted from Castellano (2013).

The construction project's work is based on the processes to be executed by the Project Team, which are organized by Project Areas from the beginning to the end of the project (Figure 3).

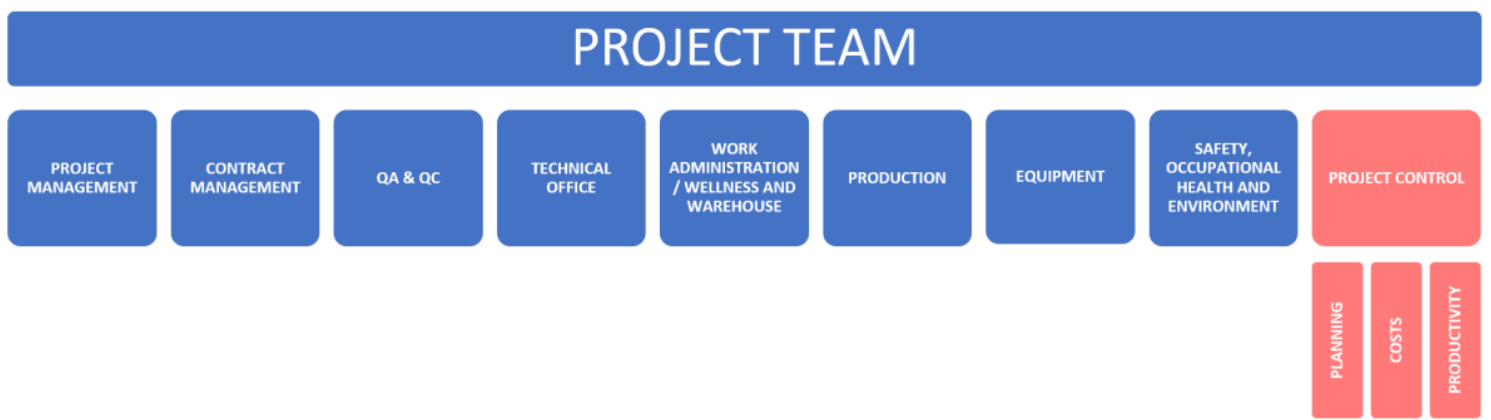

Figure 3: Structure of the work team. Adapted from COSAPI SA (2012)

\section{Construction Project Control Area}

Three areas that are part of the Project Controls (COSAPI SA, 2012): 


\section{Planning and Scheduling}

Its objective integrates, represents, and controls the project's construction planning in direct coordination with the production area and according to its requirements. The Production Area directs construction planning, while the Planning Area provides support through the development and monitoring of the General Schedule, Intermediate, and Weekly Plans, among others. Also, it is in charge of generating indicators to analyze the performance of the project deadlines.

\section{Cost Area}

Its main objective is to quantify and report project costs and margin periodically to detect deviations in the financial result concerning the updated baseline, analyze their causes and provide alerts on time for the project team to take relevant actions. The main functions of the cost area are the following: (1) Prepare the Project Phases Plan (In Cosapi, the grouping of items or related activities for their monitoring, control, and reporting is called Phase), (2) quantify costs incurred in the project, (3) prepare the projection of costs until the end of the project, in coordination with the areas of production, planning and project management and (4) integrate reports that include the sale, cost, and margin of the project to the project and the Headquarters.

\section{Productivity Area}

Its objectives are: (1) Measure the actual performance of the main construction processes of the project to their performance goals, (2) identify and propose actions to improve the performance of the main construction processes of the project and (3) document the actual performance of the construction processes considered critical by the organization and the project team, to improve feedback of the projects to the rest of the organization.

\section{The Lean Construction Professional Profile (LCPP)}

The consolidation of lean construction requires the active participation of people capable of implementing this management philosophy. For this reason, the pioneering research by Pavez and Alarcon (2007) defines a Lean Construction Professional Profile (LCPP), which identifies three areas of competence that must be developed simultaneously: Business vision, technical competence, and social competence. This research revealed the coherence of the model in terms of what construction companies expect from their project staff and how, through the identification of specific competencies, it is possible to address the three elements of lean management:

- Business purpose (business vision): It is related to understanding the strategic problems of the business and customer needs, sharing values and organizational objectives and needs of the organization.

- Processes (technical competence): It is related to construction techniques, project management, lean tools.

- People (social competence): Self-control, social skills.

For the case study of the present investigation, factors related to the LCPP are identified to select the new member for the Project Control area, as will be seen later in the application. 


\section{Choosing by Advantages (CBA)}

CBA is a complete system for consistent decision-making, including principles and definitions, models and methods, tools, and techniques. The methods it includes can be used for practically all types of decisions, both monetary and non-monetary, from simple to complex decisions (Bettler, 2010). For the present research, we used the Tabular CBA method, ideal for decisions with moderate complexity. This method can be summarized in the following seven steps (Figure 4):

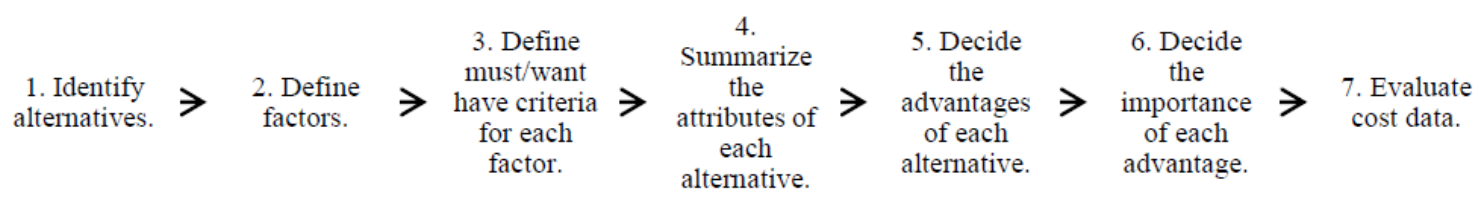

Figure 4: Steps of the CBA Tabular Method (Schöttle et al., 2015)

\section{CASE STUDY BACKGROUND}

Implementing CBA training is necessary (Schöttle \& Arroyo, 2016). Considering this critical requirement, before the application, the team proceeded to theoretical training sessions plus a practical workshop of the Tabular CBA method in person with the project team (keeping distance and using protective equipment in the current context of the pandemic). This training with members from different areas allowed us to brainstorm potential future applications in decision-making to continue consolidating the culture of making decisions using this consistent method.

In the project, the need arose to add one more member to the Project Control area team. Therefore, the new staff's required profile to join the area is generated, and a request is sent to the Human Resources area of the Central Office.

Due to fluctuations in physical permanence in work due to both the work regime and the promptness of following the recruitment and selection processes to select the new member of the area, the CBA Tabular method's application was carried out remotely. Supporting us with a collaborative virtual tool, so we proceeded to design the necessary interface and template. In this way, counting on the previous training of the Tabular CBA method's concepts and using the virtual tool containing the prepared template and the high predisposition, we proceeded to the application.

\section{Application of the Tabular CBA Method}

Step 1. Identify alternatives: A list of personnel available in the company that meets the team's indicated requirements is proposed. It is facilitated for us also to add referrals to the list. A list is made with all the identified alternatives, from which a single person will be chosen to fill the vacant position.

Step 2. Define the factors: We asked ourselves the need for the project that our Project Control area could efficiently cover with the incorporation of a person. Measurements in the field were not taking place continuously due to two relevant root causes: 1 . the work regime, which is $21 \times 7$, which left field measurements with an empty week, 2. the saturation of the workweek between the requirement for deliverables from the Central Office and the requirement for deliverables requested by the Client, allowing only one productivity measurement per week to be obtained. Also, the objective was to become a high-performance team in the shortest possible time to overcome the challenges that are approaching in the coming months when addressing the monitoring and control of Architecture and Installations activities that will be executed at home. 
After brainstorming in the team, the following factors were defined to evaluate the applicants:

- Factor 1. General previous experience. It is important to know the experience and knowledge of the company's management system that will help accelerate the learning curve in the team.

- Factor 2. Previous experience in the Project Control area. How related are Project Control's specific issues (Costs, Planning, and Productivity)? Having worked directly or indirectly with the aforementioned topics will significantly benefit the team's work and help accelerate their learning curve. Knowledge (minimally basic) of the Lean Construction philosophy, project management standards such as that provided by the Project Management Institute, Earned Value methodology, among other specifics, is essential.

- Factor 3. Inclination or affinity towards the Project Control area. It is important to know the applicant's expectations concerning the area of Project Control. The specialization in this area is aligned with the company's vision, allowing it to improve the processes according to the organization's needs. Also, suppose the professional projection is in accordance with the topics of the area. In that case, the applicant will have a greater focus and motivation to learn, develop knowledge, and seek improvements and implement them.

- Factor 4. Attitude or predisposition towards work. It will allow being up to date with the challenges to comply with the monitoring and control of the project's work.

- Factor 5. Create a good work environment. Spending 3/4 of a month living at work implies that a requirement is to facilitate a good or excellent work environment in the area. Informal conversation topics, hobbies, professional/personal goals, or other related will allow the creation of this space that allows developing a degree of affinity and trust among the team members to face the project's challenges.

- Factor 6. Software domain. Due to the processing of a massive amount of information and the creation of databases to manage them efficiently, the domain of Excel and dynamic tables are necessary for an adequate performance of Project Control and the management of Autocad for consultation metering of plans. Optional knowledge and management of other tools that allow the monitoring and updating the 3D and 4D models currently used in the project.

- Factor 7. Home location. This factor was determined due to the specific geographic context of the project. It is located approximately 10 hours from Peru's capital, with a single route and limited companies that provide transportation services and the established work regime. The applicant's location should allow an accessible movement, which does not generate inconveniences than the stipulated work hours, in addition to reducing the risk of potential contagion (in the current context of the pandemic) caused by exposure in long trips to and from the construction site. 


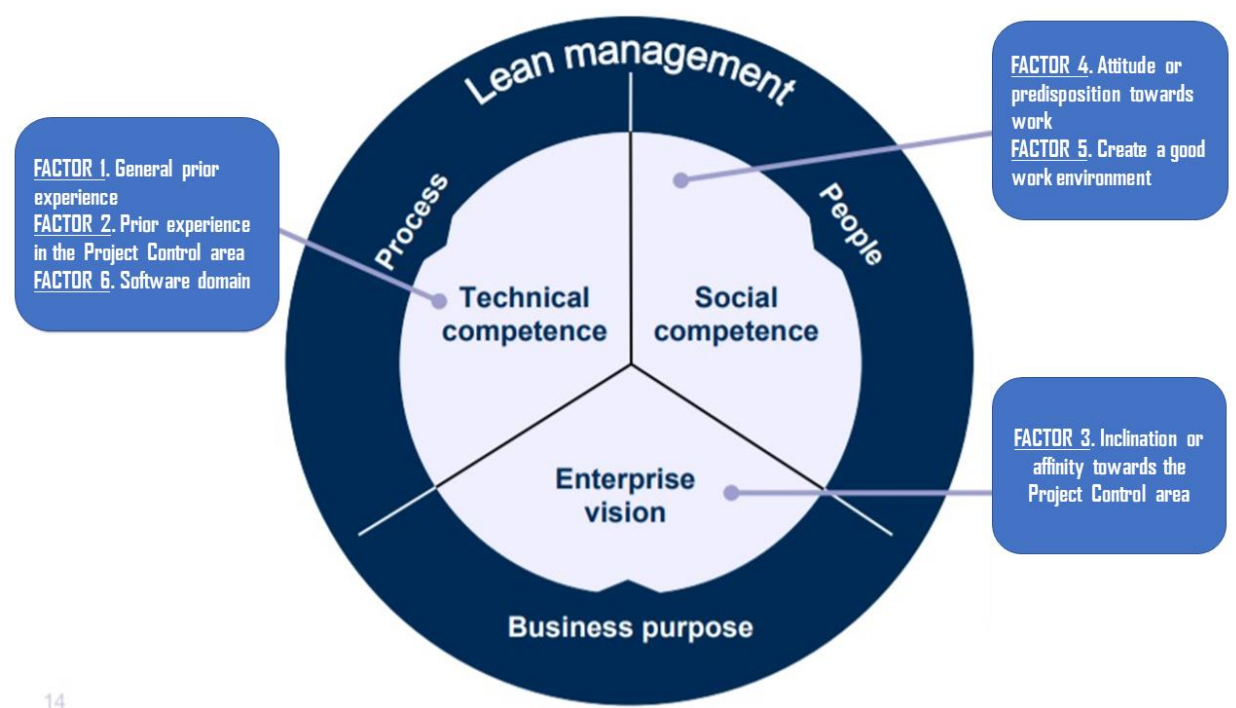

Figure 5: Factors used related to LCPP. Adapted from Pavez \& Alarcon (2007)

Step 3. Define the criteria: The guidelines that will allow routing the advantages between the alternatives' attributes are determined associated with each factor.

Step 4. Summarize each alternative's attributes: The information indicated in each of the applicants' CVs and information obtained directly from a personalized interview carried out with each of them was used as sources of the attributes. For this step, it is necessary to detail that the interview aimed to obtain the attributes according to the factors and their respective criteria identified in the previous steps. This information was fed directly to the template generated in the virtual tool after each interview was carried out.

Step 5. Decide the advantages for each alternative: After completing the interviews and completing all the alternative attributes. The team proceeded to obtain the advantages of each of them, anchoring the difference against the "worst attribute" obtained, depending on each factor's criteria. From this step forward, work was done directly on the virtual platform template collaborative and real-time.

Step 6. Decide the importance of each advantage: We find in this particular step the great support of working in a collaborative virtual platform, due to the ease of emulating reality when working with post-its, ease of moving figures, writing in time real and shared, in addition to that the video call program allows argumentation and the exchange of ideas, both tools being of great help when generating consensus.

According to Suhr (1999), there is no totally objective decision, all decisions are loaded with values and therefore it is finally necessary to decide or weigh intensities of preferences. In our application, the subjectivity regarding the importance of the advantages in our factor "Affinity for the area of Project Control" lies in our perception of the applicant based on their training information (certificate at a basic level in project control, the number of courses, seminars and workshops related to Project Control that each one attended) and the answer to questions related to Project Control. What marked the differential of advantage among the applicants in this last interview, was the responses of one of them, which showed that his desired career line was aimed at becoming a Project Control Manager. Finally, being considered by us, this advantage as the most important in our selection, it was labeled as the paramount advantage.

In this way, we determine the paramount advantage, to which we assign the maximum score of 100 (great Affinity for Project Control), and then from this, we give a score to 
each of the other advantages (in decimal scale). Finally, we add the total advantage importance score for each of the alternatives.

Step 7. Evaluate the cost information: This step was not relevant for our analysis because the applicants were in a company's salary band. In our country, for junior engineers, the salary band used in the construction market varies in an interval between approximately $10 \%$ and $15 \%$, this difference was within the limits of the project budget for the requested position. For this reason, for practical purposes, step 7 of the CBA selection was skipped. The decision was ultimately made based on the highest total advantage importance score.

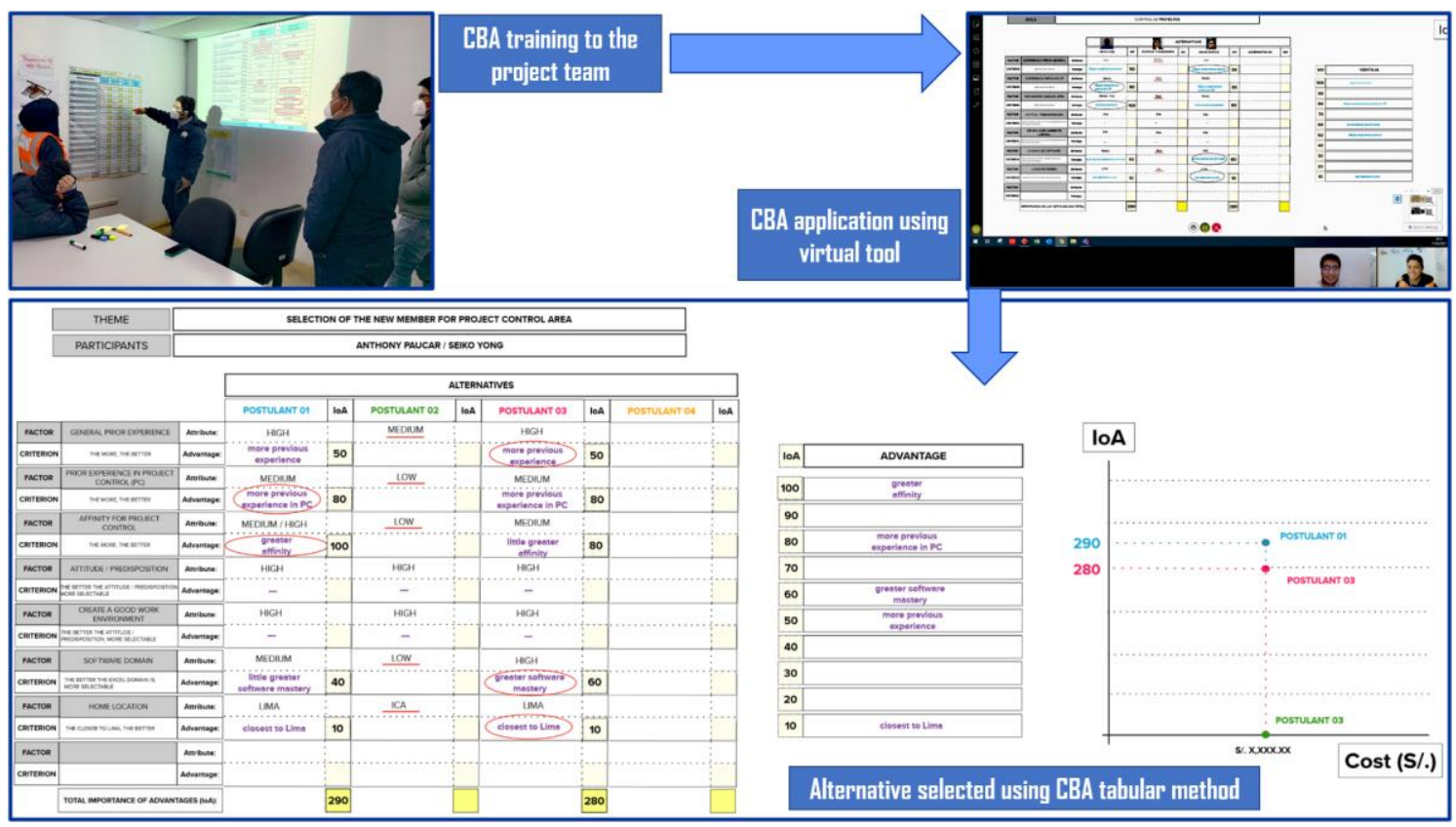

Figure 6: CBA application to select a new member of the project team.

\section{DISCUSSION}

The following table shows the matrix resulting from the application of the CBA Tabular method for our case. It is observed that applicant 02 does not have advantages over the other alternatives in any factor, obtaining a null score of the importance of advantages.

Among the factors related to technical competencies, the importance score of the advantages of the 'Software domain' factor differs due to the management of information in databases among the applicants.

The factor related to the business purpose differs in the importance score of the advantages of 'Affinity for Project Control,' which also contains the paramount advantage of the evaluation. The team's priority is that the selected applicant is found aligned to the vision of the company by belonging to the Project Control area, focusing on the search and implementation of improvements in the project.

Among the factors related to social competencies, there is no difference in the importance score of the advantages of any factor, showing that all the applicants interviewed had similar high attributes that did not generate an advantage over the other.

The 'Home location' factor contains the minor advantages scored on the importance scale with the same score for applicants 01 and 03.

Finally, the decision is made to select a new member of the Project Control area, applicant 01, with the highest total IoA score (290). 


\begin{tabular}{|c|c|c|c|c|c|c|c|c|c|c|c|}
\hline \multirow{5}{*}{ 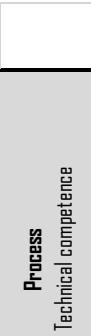 } & \multicolumn{2}{|l|}{$\begin{array}{c}\text { Factor } \\
\text { (Criterion) }\end{array}$} & \multicolumn{3}{|c|}{$\begin{array}{l}\text { Alternative 1: } \\
\text { Postulant } 01\end{array}$} & \multicolumn{3}{|c|}{$\begin{array}{l}\text { Alternative 2: } \\
\text { Postulant } 02\end{array}$} & \multicolumn{3}{|c|}{$\begin{array}{l}\text { Alternative 3: } \\
\text { Postulant } 03\end{array}$} \\
\hline & General Prior Experience & Att: & High & & & Medium & & & High & & \\
\hline & $\begin{array}{l}\text { (The more general prior experience, the } \\
\text { better.) }\end{array}$ & Adv:: & $\begin{array}{l}\text { More previous } \\
\text { experience }\end{array}$ & Imp.: & 50 & & Imp:: & & $\begin{array}{l}\text { More previous } \\
\text { experience }\end{array}$ & Imp.: & 50 \\
\hline & Prior experience in Project Control (PC) & Att: & Medium & & & Low & & & Medium & & \\
\hline & $\begin{array}{l}\text { (The more general prior experience in } \\
\text { PC, the better.) }\end{array}$ & Adv.: & $\begin{array}{l}\text { More previous } \\
\text { experiencein PC }\end{array}$ & Imp.: & 80 & & Imp:: & & $\begin{array}{l}\text { More previous } \\
\text { experience in PC }\end{array}$ & Imp.: & 80 \\
\hline & Software domain & Att: & Medium & & & Low & & & High & & \\
\hline & $\begin{array}{l}\text { (The better software domain, more } \\
\text { selectable.) }\end{array}$ & Adv:: & $\begin{array}{l}\text { Litte greater software } \\
\text { mastery }\end{array}$ & Imp.: & 50 & & Imp.: & & Greater soffware mastery & Imp.: & 60 \\
\hline \multirow{2}{*}{ 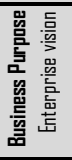 } & Affinity for Project Control & Att: & Medium / high & & & Low & & & Medium & & \\
\hline & (The more affinity for PC, the better.) & Adv:: & Greater affinity & Imp.: & 100 & & Imp.: & & Little greater affinity & Imp.: & 80 \\
\hline \multirow{6}{*}{ 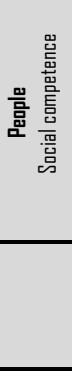 } & Attitude / predisposition towards work & Att: & High & & & High & & & High & & \\
\hline & $\begin{array}{l}\text { (The better attitude/predisposition, more } \\
\text { selectable.) }\end{array}$ & Adv:: & - & Imp.: & & - & Imp.: & & - & Imp.: & \\
\hline & Create a good work environment & Att: & High & & & High & & & High & & \\
\hline & $\begin{array}{l}\text { (The better potential for create a good } \\
\text { work environment, more selectable.) }\end{array}$ & Adv: & . & Imp.: & & - & Imp.: & & - & Imp.: & \\
\hline & Home location & Att: & Lima & & & Ica & & & Lima & & \\
\hline & (The closer to Lima, the better.) & Adv.: & Closest to Lima & Imp.: & 10 & & Imp.: & & Closest to Lima & Imp.: & 10 \\
\hline & Total of loA & & & & 290 & & & 0 & & & 280 \\
\hline
\end{tabular}

Figure 7: Constructed Case - Evaluation using CBA Tabular method.

\section{CONCLUSIONS}

Construction is a project-based industry. Each project needs work teams made up of people who will contribute with their professionalism, knowledge, and experience to guide it to its successful completion, meeting the established objectives.

This research was carried out in the context of incorporating a new member of the project team, supported by factors related to the profile of a lean professional, to perform functions in the Project Control area of a hospital building project. Taking seriously the task of finding the right person who would add to the efforts to achieve the area's objectives and the project, the CBA tabular method was applied.

We recommend designing and carrying out the previous training of the CBA system, and that it includes both developed and application examples, ranging from simple to complex decisions. This good practice allowed, in our case, to fix the concepts of the system in each of the participants, arouse interest in applying the Tabular CBA method in various selection problems. Create a predisposition to participate in collaborative decisions, and document decision-making as a knowledge asset that can be consulted later in the face of future similar selection problems in the organization.

In this study case, we indicate the support provided by technology by using a virtual collaborative platform plus a video call platform, which allowed the development of personalized interviews with each of the applicants. It permits the application of the CBA tabular method to make the selection decision (we use the collaborative platform 'Mural' and the video call platform 'Microsoft Teams'). Future research may delve into the use of digital platforms to efficiently develop a CBA method's training and application with the team. Finally, this work details the CBA application until the selection of the new member. In future research we will delve into an application that allows us to go to reconsideration, 
where new alternatives or the impact of cost on the advantages after its implementation can be analyzed.

\section{REFERENCES}

Bettler, L. (2010). "Choosing By Advantages: A very brief introduction," Accessed March 20, 2021. Slide 1 (leanconstruction.org).

Campero \& Alarcón (2003). Administración de proyectos civiles (Segunda Ed.). Ediciones Universidad Católica de Chile.

Castellano, F. (2013). Propuesta de mejora del proceso de reclutamiento y selección en una empresa de construcción e ingeniería [Proposal to improve the recruitment and selection process in a construction and engineering company]. BSc. Peruvian Universiy of Applied Science [In Spanish].

Ding, X. \& Parish, K. (2019).'A Proposed Lean Decision-Making Process for Building Energy Retrofits' In:, Proc. 27th Annual Conference of the International Group for Lean Construction (IGLC). Dublin, Ireland, 3-5 Jul 2019. pp 1357-1366.

Fong, P. S. W. \& Lung, B. W. C. (2007). Interorganizational teamwork in the construction industry. Journal of Construction Engineering and Management, 133(2), 157-168.

COSAPI S.A. (2019). Manual de Gestión de Proyectos. Gerencia de Gestión de Operaciones, Lima, Perú.

García, T. D., \& Tantalean, I. (2012). "Selección y control del factor humano en empresas de construcción civil". [Selection and control of the human factor in civil construction companies] [In Spanish] Industrial Data, 15(2), 063-072.

Lean Construction Enterprise (2011). "Equipos de Trabajo en Proyectos de Construcción." $\begin{array}{lll}\text { Accessed April 15, } 2021 . & \end{array}$ https://sites.google.com/a/leanconstructionenterprise.com/lean-constructionenterprise/documentacion/equipos-de-trabajo-en-proyectos-de-construccion.

Pavez, I. \& Alarcon, L. F. (2007). 'Lean Construction Professional's Profile (LCPP): Understanding the Competences of a Lean Construction Professional' In: Pasquire, C.L, C. L. \& Tzortzopoulos, P., 15th Annual Conference of the International Group for Lean Construction. East Lansing, Michigan, USA, 18-20 Jul 2007. pp 453-464.

Schöttle, A., Arroyo, P. \& Bade, M. (2015). 'Comparing Three Methods in the Tendering Procedure to Select the Project Team' In: Seppänen, O., González, V. A. \& Arroyo, P., 23rd Annual Conference of the International Group for Lean Construction. Perth, Australia, 29-31 Jul 2015. pp 267-276.

Schöttle, A. \& Arroyo, P. (2016). 'The Impact of the Decision-Making Method in the Tendering Procedure to Select the Project Team' In: 24th Annual Conference of the International Group for Lean Construction. Boston, Massachusetts, USA, 20-22 Jul 2016.

Suhr, J. (1999). The Choosing By Advantages Decision Making System. Quorum, Westport, CT.

Wilson, M. A. (2003). Collaborative decision making: building consensus group decisions for project success. Paper presented at PMI® Global Congress 2003-North America, Baltimore, MD. Newtown Square, PA: Project Management Institute. 